\title{
Resolutions Adopted by IAU Colloquium 135
}

During the second general discussion, held on 10 April 1992 and chaired by H. Abt, Colloquium participants discussed and approved several resolutions. The first of these was the result of a recommendation by J. Mattei and amplified by P.-L. Bernacca that the Colloquium urge the continued support of the HIPPARCOS mission. Leading to the second resolution, R. Harrington urged the need for publication of the epoch of osculation of an orbit. H. McAlister proposed a resolution regarding the nomenclature of the geometrically-determined distance to a resolved spectroscopic binary. Following a discussion prompted by R. Griffin and amended by $\mathrm{H}$. Abt, a resolution regarding orbital cycle number was adopted. Finally, remarks by E. van Dessel prompted a resolution in support of the needs of small telescopes. A resolution by R. Griffin urging the adoption of the term arc millisecond rather than milli-arcsecond was defeated.

The detailed resolutions as adopted by IAU Colloquium 135 are as follows:

Resolution I: Recognizing the quality of the results already obtained from the processing of one year of observations carried out by the ESA HIPPARCOS satellite - in particular, in the field of double star astrometry and photometry - and acknowledging the value of the scientific return provided by a long and continuous coverage of double and multiple stars, the participants of I.A.U. Colloquium 135 on Complementary Approaches to Double and Multiple Star Research recommend that the HIPPARCOS mission operations be extended to the end of the satellite lifetime.

Resolution II: Recognizing the increased precision with which multiple star observations can be made, and the increased accuracy to which multiple star ephemerides need to be calculated, and thus recognizing the need to include changing perspective effects in nearby stars, three-body effects in triple stars, and precession in all cases, it is resolved that both an epoch of osculation (usually the mean epoch) and an epoch of equator/equinox (where relevant) be included as parameters of all double star orbits.

Resolution III: The combination of $\left(a_{1}+a_{2}\right) \sin i$ from a double-lined spectroscopic binary with $a^{\prime \prime}$ and $i$ from a visually or interferometrically resolved binary gives a direct determination of the distance to a binary system for which these complementary parameters exist. With the expectation that advances in high angular resolution and precise radial velocity methods will greatly increase the applicability of this powerful means of distance determination, we resolve that the parallax found by combining $\left[\left(a_{1}+a_{2}\right) \sin i\right]$ with $\left[a^{\prime \prime}, i\right]$ be known as the orbital parallax.

Resolution IV: Recognizing that the integral part of the phase of a cyclical astronomical phenomenon should properly represent a cycle count, it is recommended that observers include the cycle number in their published tabular data, not just a fractional phase.

Resolution V: Recognizing the importance of small telescope for research in many areas in astronomy, including the study of double and multiple stars, it is urged that those with the responsibility for the operations of small telescopes take into consideration the needs for such telescopes. 